\title{
Developing Critical Thinking Skills in a Mixed-Signal Test and Product En- gineering Course
}

\section{Dr. Tina Hudson, Rose-Hulman Institute of Technology}

Tina Hudson is an Associate Professor at Rose-Hulman Institute of Technology. She received her Ph.D. in Electrical Engineering from Georgia Institute of Technology in 2000. She teaches in the areas of analog and digital circuits and systems, analog and mixed-signal integrated circuit design and testing, and MEMS. Her education research interests include the development of critical thinking skills and intuition in undergraduate students and course development based on learning theory.

\section{Mrs. Shannon M. Sipes, Rose-Hulman Institute of Technology}

Shannon M. Sipes has served as the Director of Assessment at Rose-Hulman Institute of Technology since 2004. She is a unique resource for faculty with her background in social science and education combined with experience applying it to STEM fields. Shannon holds B.S. and M.A. degrees in psychology and is currently finishing her Ph.D. in curriculum and instruction with a focus on higher education. In her current professional role, Shannon performs assessment functions at all levels from small classroom projects through assessment at the institute level. Additionally, she spends a substantial portion of her time collaborating with faculty on educational research projects and grant funded projects requiring an assessment component. Her own research interests are in inquiry methodology, gifted students, and curriculum design. 


\title{
Developing Critical Thinking Skills in a Mixed-Signal Test and Product Engineering Course
}

\begin{abstract}
Students were encouraged to follow a deep approach to learning in a Mixed-Signal Test and Product Engineering course to improve their critical thinking skills. The course used hands-on laboratory experiences containing multiple variables which effected student measurements of specifications in mixed-signal circuits. Students were required to use critical thinking skills to design tests to isolate these variables and evaluate their measurements for sufficient accuracy while minimizing testing time. The instructor modeled the critical thinking skills early in the term and provided many opportunities for the students to practice the critical thinking skills in a setting that provided immediate feedback. The improvement in critical thinking skills was measured using the Critical Thinking Skills Assessment Test developed at Tennessee Tech. A 0.95 bias effect was demonstrated between the pre- and post-test scores. A focus group with the students triangulated the results and provided suggestions as to which elements of the course the students perceived caused the most improvement in their critical thinking skills.
\end{abstract}

\section{A. Introduction}

There is an outcry from technological employers and academia to help students develop strong critical thinking skills. ${ }^{1,2,3}$ While critical thinking has different meanings to different people, there are many sources that connect critical thinking with the higher levels on Bloom's Taxonomy: comprehension, application, analysis, synthesis, and evaluation. ${ }^{3,4,5}$ Our entering student population exhibits a wide variety of abilities in these areas, and many higher education courses fail to bring them to a new level. Finding ways to improve these skills is an objective widely sought after in higher education; however, obtaining this goal is often elusive.

Felder and Brent suggest that encouraging students to take a deep approach to learning will indeed bring students to higher levels of intellectual development. ${ }^{6}$ A student taking a deep approach to learning goes beyond learning facts, procedures, and pattern matching. Instead, the student tries to understand the fundamentals underlying the formulas and procedures so that they can connect the concepts to the ideas already in their understanding. Felder also suggests that the methods that an instructor employs in the classroom can encourage students to take this deep approach to learning and thereby bring them to higher levels of intellectual development. The authors of this paper have developed a course, Mixed-Signal Test and Product Engineering, which uses many of these instructional techniques and has demonstrated a significant shift in critical thinking skills among the students.

Test Engineering is a sector of the integrated-circuit industry where engineers validate that fabrication variation does not compromise the quality of integrated-circuit performance. Test engineers write programs to automatically control test equipment to accurately and quickly test complex integrated circuit designs. Mixed-signal products integrate highly efficient digital com- 
putation with analog circuits required to interact with the "real-world." The Mixed-Signal Test and Product Engineering course presents the methods test engineers use to validate the performance of mixed-signal circuits, such as comparators, digital-to-analog converters (DACs), and analog-to-digital converters (ADCs). This course, developed in conjunction with industry, integrates material from many core courses including analog circuits, digital circuits, signal analysis, feedback control, programming skills, statistical analysis, and data analysis. Students are expected to integrate their experiences from their core courses and apply those skills in this industryfocused "real-world" application. A complete overview of the course can be found in Hudson et.

al. ${ }^{7}$ In this work, the authors will primarily focus on the elements of the course that have fostered higher levels of intellectual development and thereby critical thinking skills.

Felder and Brent ${ }^{6}$ suggest that there are five teaching conditions that correlate with students taking a deep approach to learning. The first condition, "Student-perceived relevance of the subject matter" is obtained by the industrial involvement and real-world problem solving. Since this course was co-developed by industry, it has received the "industry stamp of approval" which gives the students incentive and enthusiasm to learn the course material. The second condition, "Clear expectations, practice, and feedback" is obtained in the laboratory exercises (labs). The critical thinking process is modeled by the professor in the early labs to ensure that the students understand what is expected. The students obtain a great deal of practice in the subsequent labs. Finally, the students receive immediate feedback about their critical thinking decisions through the industry-grade automatic test equipment (ATE). "Appropriate tests" or students assessments, the third condition, is obtained by requiring deep analysis in lab, homework, and exams, all of which contribute to their grade. The forth condition, "Reasonable workload" is handled to the best of the professor's ability. Since the course is an upper-level course and not a pre-requisite for any other course, the professor can adjust the content if necessary to help the students master these higher-level thinking skills. "Choice over learning tasks," the last condition, is not adhered to as strongly as the other conditions. The final mini-project, encompassing the last three labs, allows some autonomy in choice. The mini-project is very open-ended allowing the students to choose one of many methods to obtain the desired results. Emphasis is not placed on complete success of the project, but rather the critical thinking choices and validation that led each group to their final conclusions. Further details of the types of lab exercises and homework assignments that are used to encourage this deep approach to learning is given in the following sections.

Student improvements in critical thinking skills were evaluated using the Critical Thinking Assessment Test (CAT) developed by Tennessee Tech ${ }^{8}$. Students exhibited a 0.95 bias shift between pre- and post- test scores, suggesting that the class had a significant impact on their critical thinking skills. A student focus group triangulated these results, showing that the students felt that the course improved the same aspects of their critical thinking skills as demonstrated by the CAT. Additionally, the focus group provided insight as to which elements of the course the students felt impacted their learning most significantly.

\section{B. Teaching High Levels of Intellectual Development In Lab}

The lab exercises are one of the primary methods of giving students a great deal of experience with higher-order thinking skills and critical thinking. The lab experiences emulate the experience 
of the test engineer in industry, which provides motivation for the students to be engaged with the learning opportunity and work through the challenges associated with them. The students are faced with multiple variables that could compromise their measurements, and they must use critical thinking to determine if their measurements are good and if not, which variable is compromising the measurement. They receive immediate feedback about their critical thinking hypotheses through the testing apparatus, which tells them whether or not their critical thinking was correct.

A diagram showing the software/hardware testing apparatus is shown in Fig. 1. The circuit that the students are intending to test, called a device-under-test (DUT - A in Fig. 1), is inserted into a test board, called the device-interface-board (DIB - B in Fig. 1). Depending on the lab, the DUT will be a comparator, a DAC, or an ADC. The DIB contains circuits that will allow the DUT to be placed into different configurations for different specification tests (e.g. input bias current, offset voltage, linearity, propagation delay, etc.). The DIB circuits may be as simple as load structures (output resistors and/or capacitors) or input resistors to gain up input current measurements, or as complicated as negative feedback servo-loops to force the device into a known state. Relays are activated to change the DIB circuitry for each specification test.

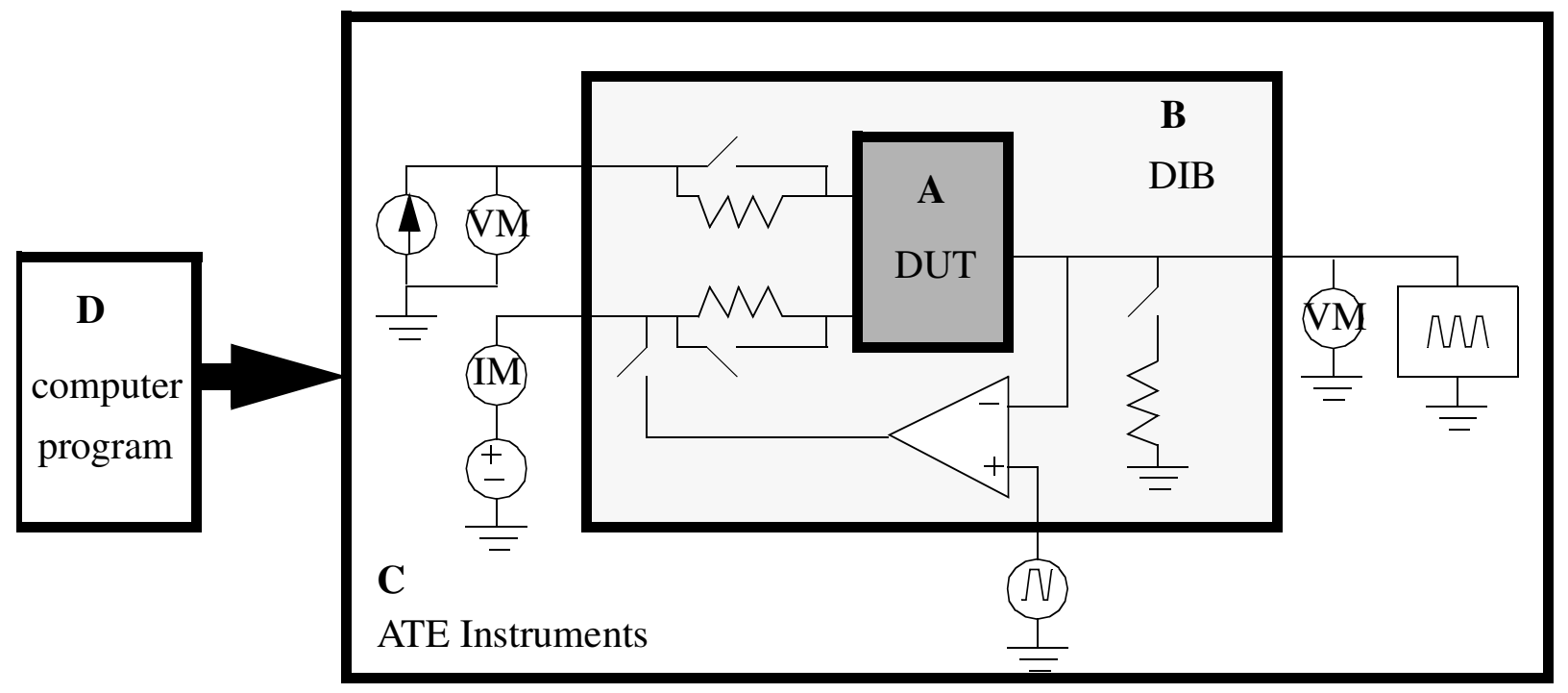

Figure 1: Diagram of test apparatus for Mixed-Signal Test and Product Engineering course lab.

Test equipment driving the DIB and DUT circuits is housed in an ATE (automatic test equipment) environment ( $\mathrm{C}$ in Fig. 1). The ATE contains analog and digital source-measurement units (units that can source current and measure voltage (VM) or source voltage and measurement current (IM)), DIB relay controls, high precision voltmeters (VM), waveform generators, and digitizers. Instrumentation activation, source values, measurement ranges, waveform choices, digitizer rates, relay control, and sequencing of events are controlled by a computer program written by the students (D in Fig. 1). The test program can also perform mathematical manipulation on the measurements to calculate DUT specifications, determine if the measured specifications meet the desired specifications (called test limits), and then indicate where the chip will be physically 
placed if the part passes or fails each of its specification tests. Fig. 2 shows a picture of the actual ATE used in the labs.

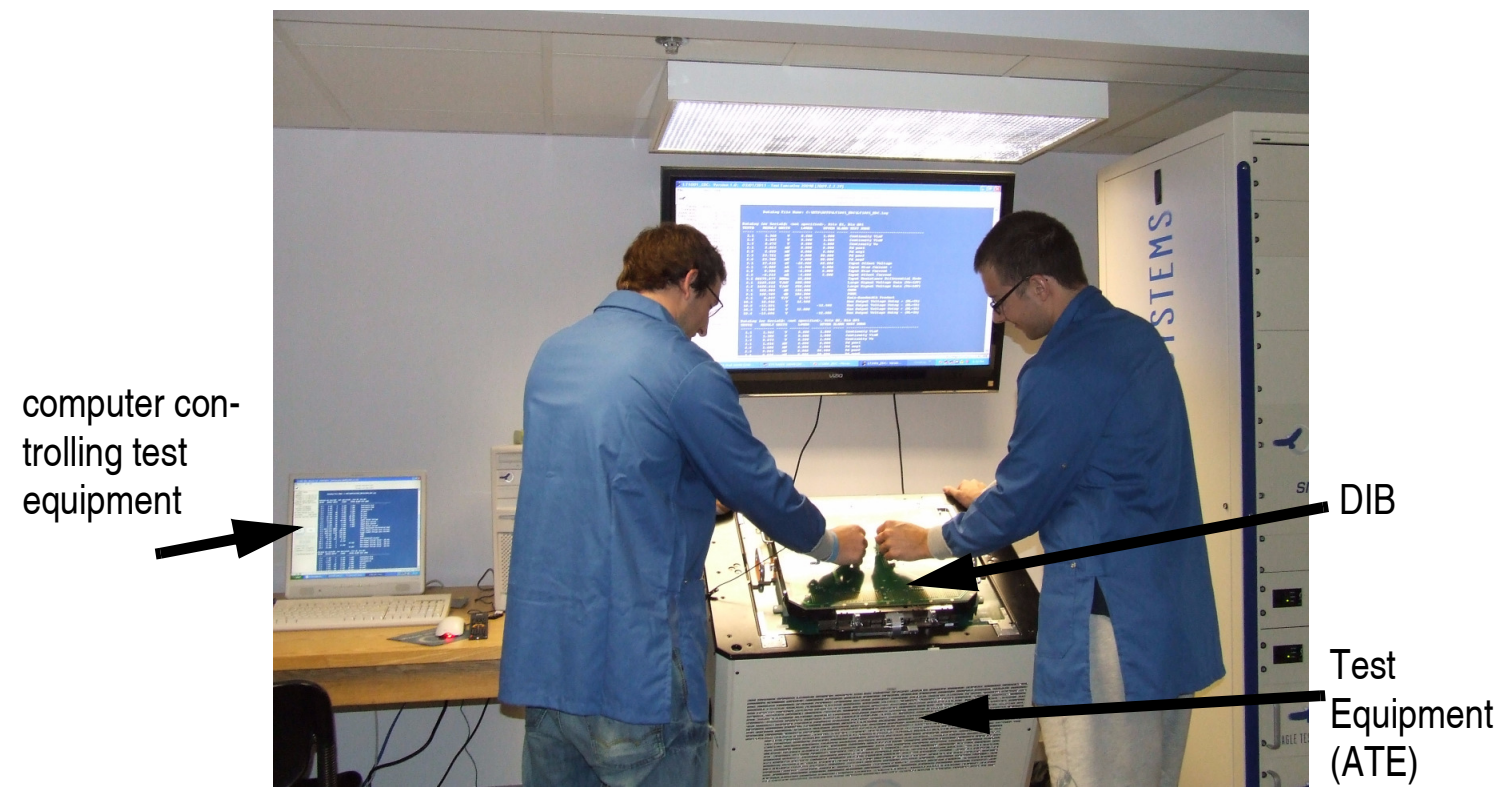

Figure 2: Picture of students using test apparatus for lab. Students are probing the DUT.

The initial labs are simple tests on a simple device (the comparator) with which the students have significant previous experience. The early labs are intended to build student knowledge of the test apparatus, familiarize them with the program environment, and give them some experience and confidence with debugging the system when there are fewer variables that may be causing incorrect measurements. However, even with the simplest test, students face many challenges and begin their training in critical thinking. An example of how this critical thinking process is emulated in the labs is described in the next paragraph.

In the first test, continuity, the students determine if the DUT was loaded into the test apparatus correctly through the presence or absence of a diode on the input and output pins. The only DIB circuits that are used in this test are relays connecting the DUT to the instrumentation. All instruments are in the same state: source-current and measure voltage. The students source a current into the diode and measure the voltage across the diode, which is a simple, robust measurement. The students have significant experience with diodes, so they know what measurement they should expect. Therefore, with this test, there are no DIB circuits to introduce measurement errors, the impact of noise is significantly reduced due to the robust measurement, there are few instrument states to introduce errors, and there are no mathematical manipulations of the measurements, which removes another source of error. As a result, the only sources of error are:

1) loading the device incorrectly,

2) controlling the DIB relays incorrectly,

3) sourcing current in the wrong direction, and

4) setting the test limits incorrectly for the measurement that is taken.

As a result, this first test has enough sources of error to require critical thinking; however, there are not so many sources of error that the student is overwhelmed. 
The first lab is validated with either the professor or an experienced student present so that the critical thinking method can be modeled for the students. When the first test is run, the students invariably get "failed" results. The professor encourages the students to look at the data to understand what is going on. When the students realize that the data shows that the diode is not being activated, they are encouraged to determine all of the reasons that this might be the case and devise an experiment to test their hypotheses.

Is the device loaded correctly? How can they determine this?

Is the source getting to the instrument pin? How can they determine this?

Is the current in the correct direction? How can they determine this?

When the students change the direction of the current, the device still fails. The professor encourages the students to again look at the data. The data correctly shows the voltage drop across a diode, indicating that the measurement is correct. Why is the test failing? The students quickly realize that the test limits are set incorrectly. Within minutes of correcting this final element, the students see correctly passing measurements, with small variability, indicating a good but robust test.

Through this first lab, the students get their first lesson in critical thinking skills: (i) What are all the possible reasons for this failure? (ii) What assumptions are being made about the system that may be incorrect? (iii) How can a test be designed to determine if the hypothesis is correct? This first lab contains many of the high-level tasks that Felder and Brent ${ }^{6}$ suggest promote a deep approach to learning. The students are required to predict the output of their experiment to justify why their data is or is not correct. The lab experiences use real data from actual devices and allow the students to test alternative explanations for the results. Students are given the opportunity to problem solve and devise experiments to test their hypotheses.

As labs progress through the quarter, the skills exhibited in the first lab are still required. However, the labs gradually introduce more conditions that can add additional measurement errors. First, more DIB circuits are included, requiring that the students question their DIB circuitry and find ways to validate whether or not the DIB circuitry is effecting their measurements. Next, a wider variety of instruments are used with more difficult modes. These modes require the students to validate that the instruments are sourcing the correct inputs before having any idea if they are getting the correct measurements. These labs require more separation of variables and the design of more experiments to test their hypotheses. Finally, labs are introduced that require measurements that are very sensitive to noise, introducing the most difficult source of error to overcome. Students are given multiple opportunities to find methods of overcoming this source of error. Since noise can be reduced by multiple methods (including averaging, waiting longer for the circuit to settle, reducing quantization noise by changing the measurement range, reducing the effect of external noise sources by changing the timing of the measurements, etc.), students are required to perform significant data analysis to determine which noise source(s) is possibly causing the poor measurement. This allows for practice with other critical thinking skills, including: (i) understanding what the data tells them about the circuit performance and (ii) interpretation of these measurements, while providing immediate feedback as to whether or not their interpretation of the data was correct. The professor/expert-student spends less and less time modeling critical thinking skills as the quarter progresses, allowing the students more opportunities to practice them in pairs, learning from one-another. 
In the final mini-project, comprising three lab periods, the students are encouraged to develop a very open-ended test on a difficult DUT with all of the measurement challenges listed previously. More emphasis is placed on the students understanding the measurement challenges and developing methods to validate their theories than getting a perfect test working. By shifting the focus of the grading, a harder problem can be posed and the students are willing to spend more time understanding their test and critically thinking about the data rather than trying anything to get it to work.

\section{Other Opportunities for Practicing Critical Thinking Skills}

While the lab is the predominate place for encouraging higher-order thinking skills, homework and exams are also places to encourage a deep approach to learning. Each DIB is specifically formulated for a given DUT; therefore the number of devices used in the lab are limited. However, in homework, the students obtain a wider experience of testing other devices through the use of datasheets.

A datasheet is a published test plan for a given device. Therefore, it is a perfect resource for the students to experience a multitude of testing methods. However, datasheets are terse and there is not a standard for them. As a result, students are required to look at multiple specifications across the datasheet to understand the testing conditions of a different specification. For example, a common-mode-rejection-ratio test (testA) may not indicate the necessary input range for the test. However, another specification, the input common-mode range, gives the maximum range that the input can go, thus giving a reasonable input change for testA. For many tests, the students must combine multiple specifications together to determine how a given specification should be tested. These types of problems give students experience determining what they know and what they do not know for a given test. Then, they must determine where else on the datasheet the additional information is located. If it cannot be found, they must make a reasonable choice given the information that they do have. Many students are very uncomfortable with this aspect of the course. Therefore, homework questions guide them to the correct locations to find the missing information early in the quarter and the students are required to justify why the information can be used. Later in the quarter, less guidance is provided.

Students are given opportunities to apply what they have learned to new situations in homework and test questions. With a multitude of datasheets available, a datasheet can be found that contains new specifications that have not been discussed in class. Students must apply their knowledge from other tests and often their knowledge from previous courses to determine the operating conditions and test procedure for the new specification.

Critical thinking skills are also practiced in homework and exams when the students design a DIB for new DUTs. Using the specification limits and operation conditions listed in the datasheet, students must design DIB components (e.g. resistor values, capacitor values, gain factors, etc.) that will successfully gain the measurements into one of several instrument ranges. These problems tend to be open-ended, with many viable solutions. Because there are many solutions due to the ranging possibilities of the instrument, students must also evaluate their component choices considering head-room for ringing and component tolerance, the impact of parasitic resistances and 
capacitances of the DIB, and the cost of the components. Much like the labs, these DIB designs increase in difficulty across the quarter, giving them simple, successful experiences early and introducing more evaluation considerations once they have mastered the early designs.

Additional practice with data analysis and evaluation skills are obtained by investigating the timeaccuracy trade-off inherent in eliminating noise. Most of the methods of reducing noise in measurements also increase test time, which has a direct relationship to the cost of the part. Therefore, students are asked to use statistical analysis techniques (Gauge Repeatability and Reproducibility studies - GRR) to evaluate their test program for this test-time versus accuracy trade-off. If the GRR evaluation is too high, students must find methods of reducing measurement variability. If the GRR evaluation is too low, students must find methods to reduce test time. Students are given exercises in GRR studies in both lab (with real data) and homework (with instructor-generated data).

\section{Evaluation of Critical Thinking Skills Improvements}

Through homework and lab exercises, students were given opportunities to develop a deep approach to learning and thereby enhance their higher-order thinking skills. To determine the degree to which students improved their higher-order thinking skills while enrolled in this course, the students were given a Critical Thinking Assessment Test (CAT) developed by Tennessee Tech

University. ${ }^{8}$ This assessment tool is a short answer essay test that measures the students' ability to evaluate information, think creatively, perform real-world problem solving, and communicate their ideas effectively. Many questions assess more than one component of critical thinking and many of these high-level critical thinking skills were assessed with multiple questions. With the short answer form of the test, all questions incorporate communication skills. This assessment exhibits a high degree of test-retest reliability and scoring reliability. ${ }^{3}$

The CAT was administered in pre-test / post-test format to a class that consisted of 9 students, including 3 juniors, 4 seniors, and 2 graduate students. One-third of the students were computer engineering majors while two-thirds were electrical engineering majors. The material in the course was covered over 10 weeks using the lectures, labs, and homework assignments described in the previous sections.

The pre-test / post-test data was evaluated for bias effect. The mean difference between the posttest and the pre-test was divided by the standard deviation of the pooled pre- and post-test groups. By dividing by the pooled standard deviation, a large change in score of a single student (an outlier) has less effect on the total bias effect calculation. Typically, a bias effect of greater than 0.5 indicates a large effect size, meaning that the course had a large effect on the post-test mean. The bias effect for the Mixed-Signal Test and Product Engineering course was 0.95. While the sample size is small, the results show promise that many of the teaching techniques employed by the instructor may have indeed had an impact on student intellectual development.

Not all questions demonstrated significant pre- to post-test gains. Moderate gains were evident in the areas of data analysis (covered under "evaluating information") and finding alternative explanations for a set of data (covered under "creative thinking"). More significant gains were found in multiple questions covered under the area of "real-world problem solving". Given that the lab 
provided many opportunities for the students to gain practice with these specific critical thinking skills, it is reasonable to assume that the course may have had an impact on the students' abilities with these skills.

In addition to the CAT assessment, the students were asked to participate in a focus group at the end of the quarter to assess their perceptions of their experiences in the course. The focus group was led by an independent facilitator who kept student responses confidential so that the students would not feel pressured to respond in a particular manner. Four students attended the focus group. Before the focus group began, the facilitator asked the students to self-rate the degree to which the Mixed-Signal Test and Product Engineering course improved specific areas of their critical thinking using a 4-point Likert scale. Students were asked to do this before the focus group started so that the focus group would not influence their ratings. Three areas were rated the highest, a 3.75/4.0. These areas included:

(i) Identify alternative interpretations for data or observations,

(ii) Integrate information to solve real-world problems, and

(iii) Learn and apply new information to solve real-world problems.

These areas directly mapped to the areas that received the most significant gains on the CAT test. The students' perceptions reinforced the hypothesis that the course was responsible for the gains on the CAT assessment.

In the focus group, students were asked to discuss specifically how the course helped them to enhance their critical thinking skills. The students unanimously reported that practicing their critical thinking skills during the testing experiences in lab provided the greatest gain in their critical thinking abilities. The students valued the fact the labs did not work the first time and that they had to struggle through multiple solutions before finding the answer. They felt these experiences allowed "other ideas to click." Students felt the labs required "more creativity and confidence than other labs" in other courses. They also valued the "unorthodox" approach that the instructor did not already know the exact solution to the lab and found it particularly effective when the instructor modeled her debugging audibly so that they were able to gain insight into her critical thinking process.

\section{Conclusions}

By developing student activities that encouraged students to take a deep approach to learning, critical thinking skills demonstrated a large improvement. While the sample size was small, the results suggest that further study is warranted. Surprisingly, students reported an appreciation for having lab experiences that required them to struggle through multiple solutions because these experiences required them to use higher-order thinking skills. While they appreciated the instructor modeling critical thinking skills, they valued the fact that the instructor did NOT have all the answers. By the end of the quarter, they were comfortable with an open-ended problem and enjoyed the creativity required to solve the problem. These student perceptions suggest that the students were moved to a higher level of intellectual development as defined by Magolda 9 . The students were exhibiting ownership for their learning, intellectual curiosity about how things interrelate, and a desire to seek out alternative explanations and find methods to understand the system better. These attributes are strongly correlated with how engineers and scientist think, 
which suggest that these types of teaching activities can have a significant impact on student intellectual development.

\section{Acknowledgements}

The authors would like to thank NSF for sponsoring this work through the Transforming Undergraduate Education in STEM (TUES) division under grant number DUE-1140363. Additionally, the authors would like to thank NSF for sponsoring the expanded use of the CAT instrument under grant number DUE-1022789. This grant allowed the Center for Assessment and Improvement of Learning at Tennessee Tech to collaborate with the authors and produce this work. The synergy that resulted from this collaboration was essential in helping the instructor improve the instructional materials and assess the results of her work.

\section{References}

[1] D. Bok, Our Underachieving Colleges: A candid look at how much students learn and why they should be learning more. Princeton: Princeton University Press, 2006.

[2] E. Halpern, “Assessing the Effectiveness of Critical-Thinking Instruction," Journal of General Education, vol. 42., pp 251-255, 1993.

[3] Stein, B., Haynes, A., Redding, M., "Project CAT: Assessing Critical Thinking Skills," in D. Deeds \& B. Callen (eds.), Proceedings of the National STEM Assessment Conference. NSF, 2007.

[4] Jessop, J.L.P, “Expanding our students' brainpower: idea generation and critical thinking skills," Antennas and Propagation Magazine, IEEE, vol. 44, (6), pp. 140 - 144, 2002.

[5] Stein, B., Haynes, A., Redding, M., Ennis, T., Cecil, M., "Assessing Critical Thinking in STEM and Beyond," in M. Iskander (ed.), Innovations in E-learning, Instruction Technology, Assessment, and Engineering Education. Springer, pp. 79-82, 2007.

[6] Felder, R. M., Brent, R., “The Intellectual Development of Science and Engineering Students. Part 2: Teaching to Promote Growth," Journal of Engineering Education, vol. 93, (4), pp. 279-291, 2004.

[7] T.A. Hudson, B. Copeland, and D. Solomon, "Creating a Mixed-Signal Test and Product Engineering Course," Proceedings of the IEEE International Conference of Microelectronics Systems, pp. 56-59, 2011.

[8] Critical Thinking Assessment Test, found at: http://www.tntech.edu/cat/home/

[9] Baxter Magolda, M.B., Knowing and Reasoning in College, San Francisco: Jossey-Bass, 1992. 